\title{
Decoherence as a Signature of an Excited State Quantum Phase Transition in Two Level Boson Systems
}

\author{
J. E. García-Ramos*, J. M. Arias ${ }^{\dagger}$, J. Dukelsky**, P. Pérez-Fernández ${ }^{\dagger}$ and \\ A. Relaño** \\ ${ }^{*}$ Departamento de Fisica Aplicada, Universidad de Huelva, 21071 Huelva, Spain \\ ${ }^{\dagger}$ Departamento de Fisica Atómica, Molecular y Nuclear, Facultad de Física, Universidad de \\ Sevilla, Apartado 1065, 41080 Sevilla, Spain \\ ${ }^{* *}$ Instituto de Estructura de la Materia, CSIC, Serrano 123, E-28006 Madrid, Spain
}

\begin{abstract}
We analyze the decoherence induced on a single qubit by the interaction with a two-level boson system with critical internal dynamics. We explore how the decoherence process is affected by the presence of quantum phase transitions in the environment. We conclude that the dynamics of the qubit changes dramatically when the environment passes through a continuous excited state quantum phase transition. If the system-environment coupling energy equals the energy at which the environment has a critical behavior, the decoherence induced on the qubit is maximal and the fidelity tends to zero with finite size scaling obeying a power-law.
\end{abstract}

Keywords: Quantum decoherence, quatum phase transition, excited state quatum phase transition PACS: 03.65.Yz, 05.70.Fh, 64.70.Tg

Real quantum systems always interact with the environment. This interaction leads to decoherence, the process by which quantum information is degraded and purely quantum properties of a system are lost [1].

The connection between decoherence and environmental quantum phase transitions has been recently investigated in [2]. In this contribution and in reference [3] we analyze the relationship between decoherence and an environmental excited state quantum phase transition (ESQPT).

An ESQPT is analogous to a standard quantum phase transition (QPT), but taking place in some excited state of the system, which defines the critical energy $E_{c}$ at which the transition takes place. We can distinguish between different kinds of ESQPT, either first order or continuous [4]. In this contribution we will concentrate in the latter case, which usually entails a singularity in the density of states (for an illustration see Fig. 1).

These kinds of phase transitions have been identified in the Lipkin model, in the interacting boson model (IBM), and in more general boson or fermion two-level pairing Hamiltonians (for a complete discussion, including a semiclassical analysis, see [5]).

Here, we consider an environment having both QPTs and ESQPTs coupled to a single qubit. The Hamiltonian of the environment, defined as a function of a control parameter $\alpha$, presents a QPT at a critical value $\alpha_{c}$. We define a coupling between the central qubit and the environment that entails an effective change in the control parameter, $\alpha \rightarrow \alpha^{\prime}$, making the environment to cross the critical point if $\alpha^{\prime}>\alpha_{c}$. Moreover, the coupling also implies an energy transfer to the enviroment $E \rightarrow E^{\prime}$, and therefore it can also make

CP1165, Nuclear Structure and Dynamics '09

edited by M. Milin, T. Nikšić, D. Vretenar, and S. Szilner

c) 2009 American Institute of Physics 978-0-7354-0702-2/09/\$25.00 


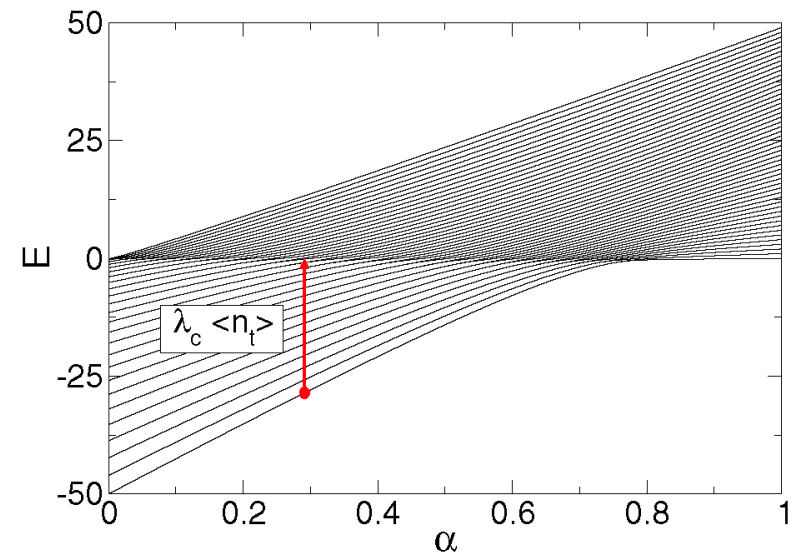

FIGURE 1. Energy levels for the environment Hamiltonian (3) with $L=0$ and $N=50$. The arrow shows the jump that the coupling with the central qubit produces in the environment.

the environment to reach the critical energy $E_{c}$ of an ESQPT.

Following [2] we will consider our system composed by a spin $1 / 2$ particle coupled to a bosonic environment by the Hamiltonian $H_{S E}$ :

$$
H_{S E}=I_{S} \otimes H_{E}+|0\rangle\left\langle 0\left|\otimes H_{\lambda_{0}}+\right| 1\right\rangle\langle 1| \otimes H_{\lambda_{1}}
$$

where $|0\rangle$ and $|1\rangle$ are the two components of the spin $1 / 2$ system, and $\lambda_{0}, \lambda_{1}$ the couplings of each component to the environment. The three terms $H_{E}, H_{\lambda_{0}}$ and $H_{\lambda_{1}}$ act on the Hilbert space of the environment.

With this kind of coupling, the environment evolves with an effective Hamiltonian depending on the state of the central spin $H_{j}=H_{E}+H_{\lambda_{j}}, j=0,1$. If the environment is initially in its ground state $\left|g_{0}\right\rangle$, the decoherence factor is determined, up to an irrelevant phase factor, by $H_{1}$, and its absolute value is equal to

$$
|r(t)|=\left|\left\langle g_{0}\left|e^{-i H_{1} t}\right| g_{0}\right\rangle\right|
$$

A value of $|r(t)|$ equal to zero implies that the qubit is no longer in a superposition of states $|0\rangle+|1\rangle$.

To be specific, let us consider a two level boson Hamiltonian, constructed out of scalar bosons, $s$, in the lowest level and bosons carrying an arbitrary angular momentum $L$ in the upper level.

$$
H_{E}=\alpha n_{L}-\frac{1-\alpha}{N} Q^{\chi} \cdot Q^{\chi}, \text { with } Q_{\mu}^{\chi}=s^{\dagger} L+L^{\dagger} s+\chi\left[L^{\dagger} \times \tilde{L}\right]_{\mu}^{(L)},
$$

where $n_{L}$ is the number of $L$ bosons, $N$ the total number of bosons and - stands for the scalar product.

This Hamiltonian has a second order QPT at $\alpha_{c}=4 / 5$ for $\chi=0$ [6], while experiences a first order phase transition for $\chi \neq 0$. We will focus in the case of $\chi=0$. Using the coherent state formalism [6] it can be shown that for $\alpha>4 / 5$ the environment 


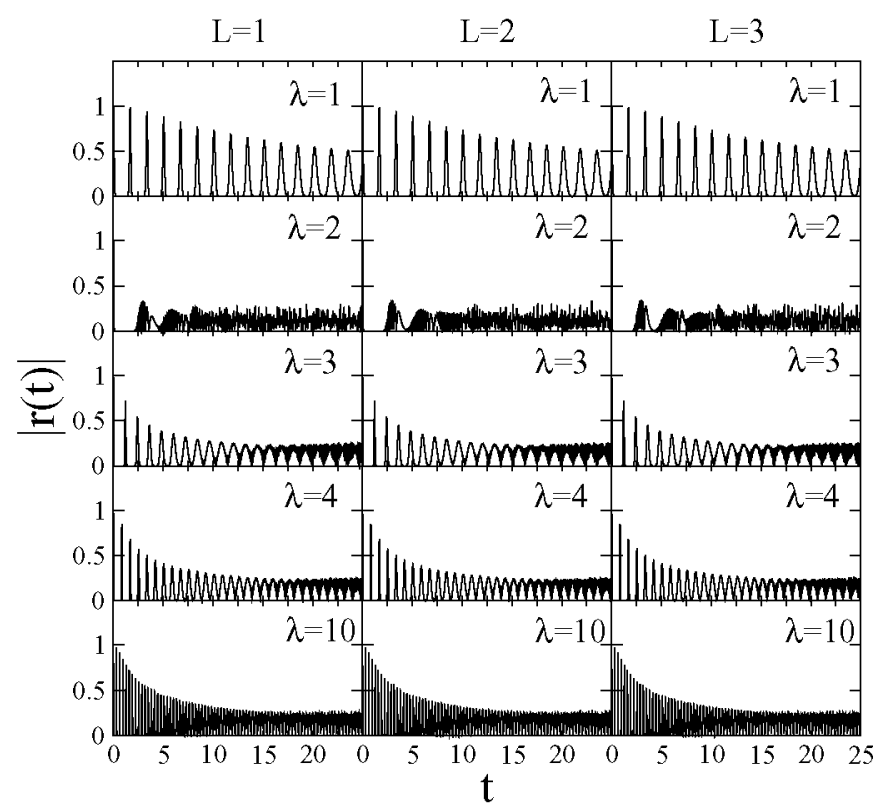

FIGURE 2. $|r(t)|$ for $\alpha=0$, five different values of $\lambda$ and $L=1,2,3$. In all cases $N=1000$.

is a condensate of $s$ bosons corresponding to a symmetric phase. For $\alpha<4 / 5$ the environment condensate mixes $s$ and $L$ bosons forming a non-symmetric phase.

Choosing $\lambda_{0}=0$ and $\lambda_{1}=\lambda$ the coupling Hamiltonian reduces to a very simple form $H_{\text {Coup }}=\lambda n_{L}$, which results into the effective Hamiltonians for each component of the systems $H_{0}=H_{E}$ and $H_{1}=H_{E}(\alpha \rightarrow \alpha+\lambda)$. Therefore, the system-environment coupling parameter $\lambda$ modifies the environment Hamiltonian. It is straightforward to show that $H_{1}$ goes through a second order QPT at $\lambda_{*}=4-5 \alpha$, for $\alpha<4 / 5$, using [6]. Furthermore, a semiclassical calculation [5] shows that $H_{E}$ also passes through an ESQPT at $E_{c}=0$, if $\lambda<\lambda_{*}$. This phenomenon is illustrated in Fig. 1.

We start the evolution with the ground state of the environment $\left|g_{0}\right\rangle$. At $t=0$ we switch on the interaction between the system and the environment, and let the system evolve under the complete Hamiltonian. By instantaneously switching on this interaction, the energy of the environment increases, and its state gets fragmented into a region with average energy equal to $E=\left\langle g_{0}\left|H_{1}(\alpha)\right| g_{0}\right\rangle$. Therefore, if $\left\langle g_{0}\left|H_{1}(\alpha)\right| g_{0}\right\rangle=0$, the coupling with the central qubit induces the environment to jump into a region around the critical energy $E_{c}$. This is illustrated in Fig. 1. Starting from a state in the non-symmetric phase with $\alpha<\alpha_{c}$, the coupling with the qubit, $H_{\lambda_{1}}=\lambda n_{L}$ increases the energy of the environment up to the critical point $E_{c}$. Resorting to the coherent state approach [6], we can obtain a critical value of the coupling strength

$$
\lambda_{c}(\alpha)=\frac{1}{2}(4-5 \alpha), \quad \alpha<\frac{4}{5}
$$

In Fig. 2 we show the modulus of the decoherence factor $|r(t)|$ for $\alpha=0$ and several 
values of $\lambda$ and $L$, corresponding to the vibron $(L=1)$, the IBM $(L=2)$ and the octupole model $(L=3)$. First, we note that the presented behavior is independent on $L$. In four of the five cases of $\lambda$ we can see a similar pattern, fast oscillations plus a smooth decaying envelope. The most striking feature of Fig. 2 is the panel corresponding to $\lambda=2$, for which $|r(t)|$ quickly decays to zero and then randomly oscillates around a small value. We note that this particular case constitutes a singular point for both the shape of the envelope of $|r(t)|$ and the period of its oscillating part. Making use of Eq. (4) for $\alpha=0$ we obtain precisely $\lambda_{c}=2$, the value at which the coherence of the system is completely lost. Therefore, the existence of an ESQPT in the environment has a strong influence on the decoherence that it induces in the central system. We can summarize this result with the following conjecture:

If the system-environment coupling drives the environment to the critical energy $E_{c}$ of a contimuous ESQPT, the decoherence induced in the coupled qubit is maximal.

This conjecture has been checked for different values of $\alpha<\alpha_{c}=4 / 5$ obtaining in all the cases that the rapid decay to zero of $|r(t)|$ always happens for $\lambda \approx \lambda_{c}$ (see Fig. 3 of reference [3]). It has been also checked how this magnitude behaves in the thermodinamical limit. The results displayed in Fig. 4 of reference [3] confirm that the presence of an ESQPT in the environment spectrum is clearly signaled by the qubit decoherence factor. Moreover, it can be defined an order parameter for the ESQPT related to $|r(t)|$.

To summarize, our main finding is that the decoherence is maximal when the systemenvironment coupling introduces in the environment the energy required to undergo a continuous ESQPT and that this results is independent on the value of $L$.

This work has been partially supported by the Spanish MEC (FEDER) under projects number FIS2006-12783-C03-01, FPA2007-63074 and FIS2008-04189, by Comunidad de Madrid and CSIC under project 200650M012, by Junta de Andalucía under projects FQM160, FQM318, P05-FQM437 and P07-FQM-02962 and by the Spanish ConsoliderIngenio 2010 Programme CPAN (CSD2007-00042). A.R. is supported by the Spanish program "Juan de la Cierva", and P. P-F., by a grant from the Spanish MEC.

\section{REFERENCES}

1. W. H. Zurek, Rev. Mod. Phys. 75, 715 (2003); M. Nielsen and I. Chuang, Quantum Computation and Quantum Information (Cambridge University Press, Cambridge, UK, 2000).

2. H. T. Quan, Z. Song, X. F. Liu, P. Zanardi, and C. P. Sun, Phys. Rev. Lett. 96, 140604 (2006); F. M. Cucchietti, S. Fernandez-Vidal, and J. P. Paz, Phys. Rev. A75, 032337 (2007); C. Cormick and J. P. Paz, Phys Rev. A77, 022317 (2008).

3. A. Relaño, J.M. Arias, J. Dukelsky, J.E. García-Ramos, and P. Pérez-Fernández, Phys. Rev. A78, 060102R (2008).

4. P. Cejnar, S. Heinze, and M. Macek, Phys. Rev. Lett. 99, 100601 (2007).

5. M. A. Caprio, P. Cejnar, and F. Iachello, Ann. Phys. 323, 1106 (2008).

6. J. Vidal, J. M. Arias, J. Dukelsky, J. E. García-Ramos, Phys. Rev. C73, 054305 (2006); J. M. Arias, J. Dukelsky, J. E. García-Ramos, and J. Vidal, Phys. Rev. C75, 014301 (2007). 